\title{
Impact of local flow haemodynamics on atherosclerosis in coronary artery bifurcations
}

\author{
Antonios P. Antoniadis ${ }^{1}$, MD, PhD; Andreas A. Giannopoulos ${ }^{1}$, MD; Jolanda J. Wentzel², PhD; \\ Michael Joner ${ }^{3}, \mathrm{MD}$; George D. Giannoglou ${ }^{4}, \mathrm{MD}, \mathrm{PhD}$; Renu Virmani ${ }^{3}, \mathrm{MD}$; \\ Yiannis S. Chatzizisis ${ }^{1 *}, \mathrm{MD}, \mathrm{PhD}, \mathrm{FESC}$ \\ 1. Cardiovascular Division, Brigham and Women's Hospital, Harvard Medical School, Boston, MA, USA; 2. Biomechanics \\ Laboratory, Thoraxcenter, Erasmus MC, Rotterdam, The Netherlands; 3. CVPath Institute, Gaithersburg, MD, USA; \\ 4. Cardiovascular Engineering and Atherosclerosis Laboratory, AHEPA University Hospital, Aristotle University Medical School, \\ Thessaloniki, Greece
}

The complete references and the accompanying supplementary data are published online at: http://www.pcronline.com/eurointervention/V_issue/4

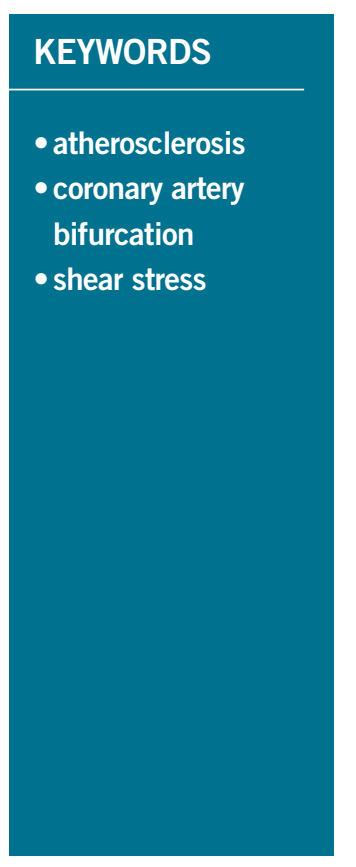

\begin{abstract}
Coronary artery bifurcations are susceptible to atherosclerosis as a result of the unique local flow patterns and the subsequent endothelial shear stress (ESS) environment that are conducive to the development of plaques. Along the lateral walls of the main vessel and side branches, a distinct flow pattern is observed with local low and oscillatory ESS, while high ESS develops at the flow divider (carina). Histopathologic studies have shown that the distribution of plaque at bifurcation regions is related to the local ESS patterns. The local ESS profile also influences the outcome of percutaneous coronary interventions in bifurcation lesions. A variety of invasive and non-invasive imaging modalities have enabled 3D reconstruction of coronary bifurcations and thereby detailed local ESS assessment by computational fluid dynamics. Highly effective strategies for treatment and ultimately prevention of atherosclerosis in coronary bifurcations are anticipated with the use of advanced imaging and computational fluid dynamic techniques.
\end{abstract}

\footnotetext{
*Corresponding author: Cardiovascular Division, Brigham and Women's Hospital, Harvard Medical School, 75 Francis Street, Boston, MA, 02115, USA. E-mail: ychatzizisis@icloud.com
} 


\section{Introduction}

Although the coronary arterial tree is uniformly exposed to systemic risk factors, atherosclerotic lesions occur at focal points. The majority of lesions develop in bifurcations, in the vicinity of the ostia of the branches and in the inner aspect of curvatures ${ }^{1}$. If they progress, they can extend into the regions at the outer curve or at the carina of a bifurcation ${ }^{2}$. Coronary artery bifurcations are susceptible to atherosclerosis as a result of the unique local flow conditions and the local endothelial shear stress (ESS) environment that develop at these locations ${ }^{3}$. Anatomic features of coronary bifurcations such as the presence and severity of plaques in the main and side branches, the diameter of the main and side branches, and the bifurcation angle all impact on local flow patterns. The interplay among the coronary anatomy, local flow and vascular biology dictates the progression and complexity of native atherosclerosis in coronary bifurcations (Online Figure 1). Coronary bifurcations are also a frequent target of percutaneous therapies, which account for 15 to $20 \%$ of all percutaneous coronary interventions ${ }^{4}$. The complex local haemodynamic microenvironment after bifurcation stenting also influences in-stent restenosis, thrombosis and clinical outcomes ${ }^{5}$.

In this review, we summarise the current data with respect to the localisation of atherosclerosis in coronary artery bifurcations and analyse the impact of flow-related local haemodynamic conditions in the initiation and progression of atherosclerosis at bifurcations. Furthermore, we present the state-of-the-art imaging modalities for invasive and non-invasive assessment of local haemodynamics in coronary bifurcations. We conclude with the implications of local ESS on clinical outcomes following percutaneous coronary interventions at bifurcations.

\section{Flow distribution in coronary artery bifurcations}

Arterial bifurcations are known for their distinct flow velocity patterns resulting in flow separation, recirculation and secondary flow patterns leading to local low and oscillatory ESS along the lateral walls (Figure 1) ${ }^{3,6,7}$. On the other hand, high ESS develops in the flow divider (carina) of the bifurcation. The proportion of flow directed towards the side branch is the primary factor determining the ESS patterns within the bifurcation (Moving image 1). Computational studies have described the patterns of flow and the impact of anatomic variations in the flow profile in coronary bifurcations $^{8}$. Furthermore, in vitro and computational fluid dynamic studies have shown that the bifurcation angle and diameter influence the local ESS patterns, i.e., the higher the angle and diameter the lower the ESS 9 .

Further to the spatial variations of ESS in coronary bifurcations, temporal alterations in ESS occur because of flow pulsatility ${ }^{10}$. During systole, ESS is low and oscillatory, while in diastole it rapidly increases up to a maximum value and then slowly declines. Flow pulsatility further aggravates the haemodynamic patterns encountered in bifurcations, potentially contributing to the initiation and progression of atherosclerosis $\mathrm{s}^{3,11}$.

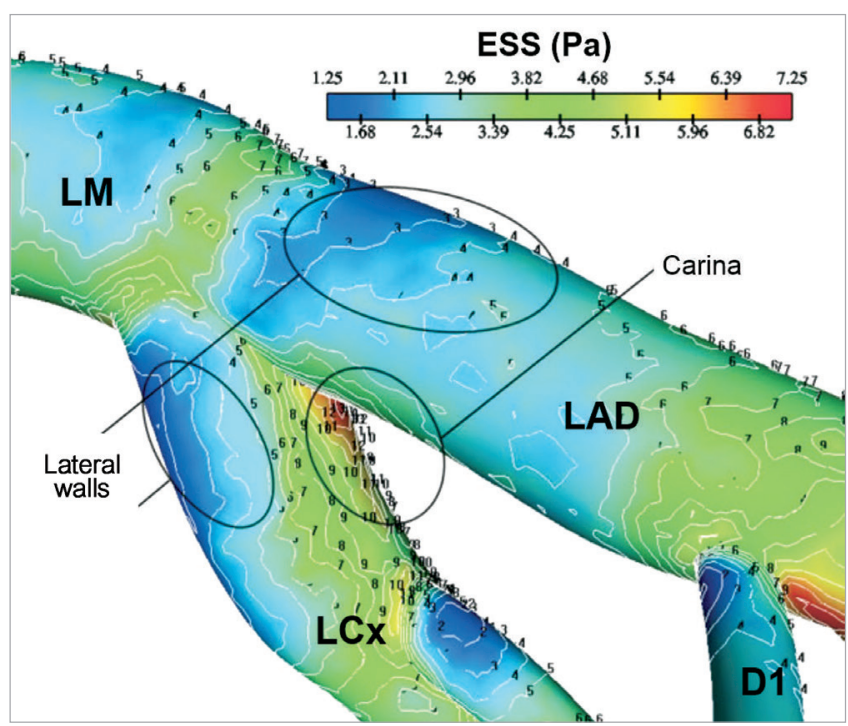

Figure 1. ESS distribution in coronary bifurcation depicting low values at the lateral walls and high values at the carina. (Reprinted with permission from Soulis et $\mathrm{al}^{7}$ )

\section{Histopathology of atherosclerosis in native and stented coronary artery bifurcations}

Low ESS modulates the molecular, cellular and vascular dynamics, which are responsible for atherogenesis and progression of plaque towards a high-risk phenotype ${ }^{12-14}$. Histopathologic studies have shown that the distribution of plaque at bifurcation regions is determined by the local ESS patterns (Figure 2A, Figure 2B) ${ }^{15,16}$. Early intimal thickening occurs in areas of low and oscillatory ESS within coronary bifurcations. Similarly, there is a higher preponderance for advanced atherosclerotic plaques with necrotic core in the low ESS areas of coronary bifurcations ${ }^{17}$. More specifically, the lateral walls (low ESS areas) have a significantly higher prevalence of plaque compared to the flow divider regions (high ESS areas) ${ }^{16}$. Interestingly, plaque might influence the local geometry by inducing lumen obstruction with subsequent flow acceleration. This may explain the observation that plaque can also be found at high ESS areas in bifurcations ${ }^{8}$.

Besides native coronary bifurcations, stented bifurcation lesions exhibit an increased frequency of atherosclerosis-associated complications (Figure 2C, Figure 2D, Figure 2E) ${ }^{16,18}$. Pathologic studies in swine have shown that eccentric neointimal hyperplasia occurs predominantly at the lateral wall of the stented main vessel of a coronary bifurcation, with concomitant acute adhesion and accumulation of leukocytes, whereas the flow divider is almost completely free of leukocytes ${ }^{19}$. Likewise, in bifurcation stenting there is an increased rate of restenosis with bare metal stents and a higher risk of late stent thrombosis with drug-eluting stents ${ }^{18}$. Notably, there are differences in the healing patterns in drug-eluting stents vs. bare metal stents. Also, in drug-eluting stents, uncovered struts and fibrin deposition are significantly greater at flow divider sites compared to the lateral walls. In this regard, coronary bifurcation stenting represents a worstcase scenario for detecting delayed vascular healing in drug-eluting 


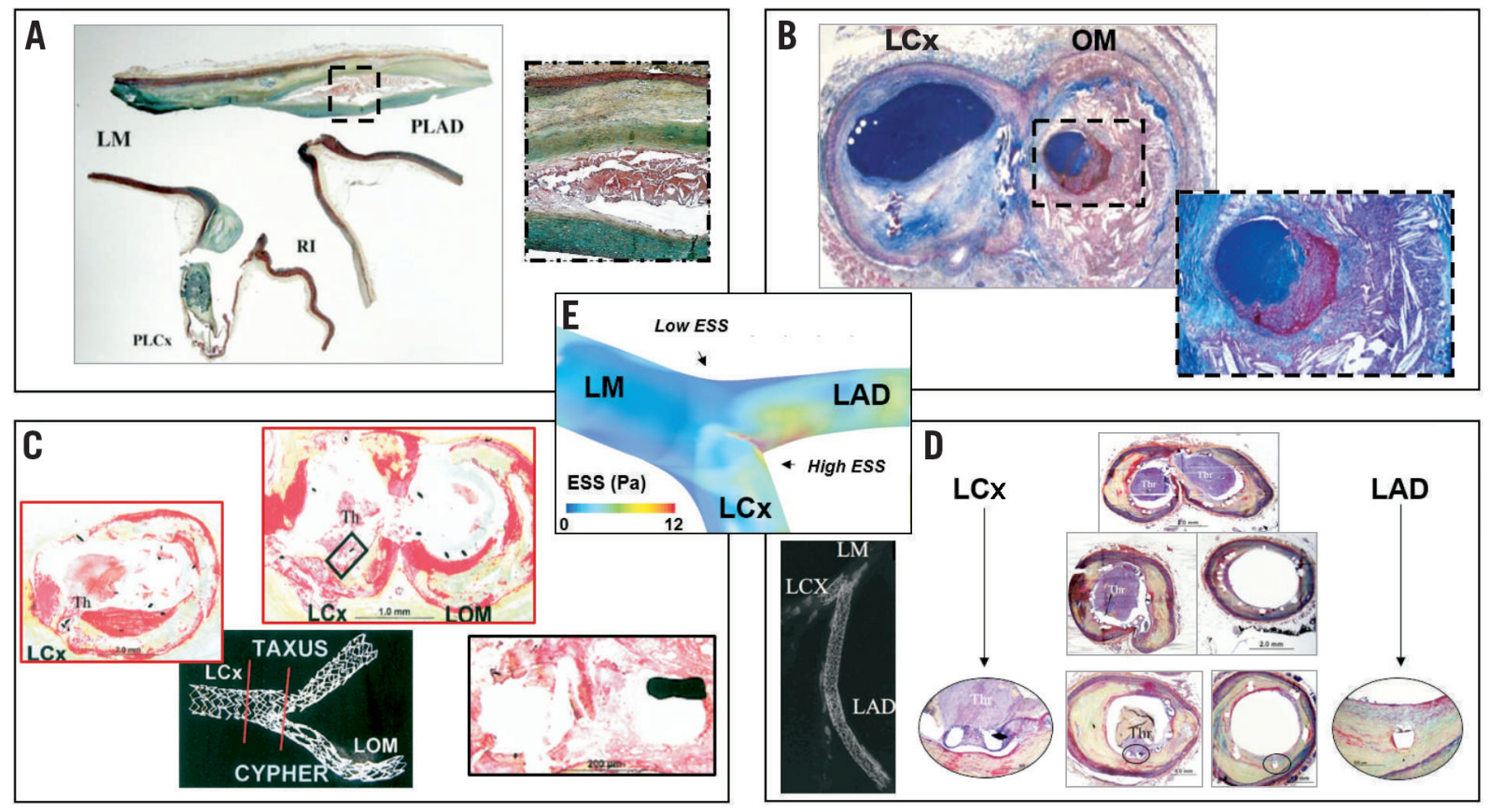

Figure 2. Representative histologic images of coronary plaque in native and stented bifurcation lesions. A) Longitudinal section taken in the region of left main (LM)-left anterior descending (LAD)-left circumflex (LCX) bifurcation showing necrotic core accompanied by heavy calcification within the plaque at the low shear regions (lateral wall), whereas the high shear (carina) has minimal intimal thickening. (Reprinted from Nakazawa et al ${ }^{16}$ with permission from Elsevier) B) Cross-section of a coronary artery bifurcation showing atherosclerotic lesions at the lateral walls, i.e., a calcified fibroatheroma on the mother branch (left) and a ruptured thin-cap fibroatheroma on the daughter branch (right and insert in zoom). (Reprinted from Schaar et al ${ }^{15}$ with permission from Oxford University Press) C) Cross-section of late stent thrombosis in ostial and bifurcation stenting showing thrombus presence (LCX) and neointimal formation (obtuse marginal [OM]). Insert zooms on two struts with overlying thrombus and absence of neointimal coverage. (Reprinted from Joner et al ${ }^{18}$ with permission from Elsevier) D) Cross-sectional images of late stent thrombosis across the course of an LAD-LCx bifurcation. Thrombus presence in the region of the uncovered struts at the flow divider (top) and in all sections of the LCX stent (left). The middle to distal portion of the LAD stent shows absence of thrombus and healed luminal surface with mild neointimal thickening. (Reprinted from Nakazawa et al ${ }^{16}$ with permission from Elsevier) E) 3D ESS distribution on a bifurcation region depicting low values at the lateral walls and high ESS at the flow divider.

stents because the inherent antiproliferative nature of the drug-eluting stents becomes amplified by local ESS-induced variations in neointimal coverage between the flow divider and the lateral wall of bifurcations. Main vessel restenosis occurs more frequently in bare metal stents as compared to drug-eluting stents, and main vessel late stent thrombosis ( $>30$ days) is greater in drug-eluting stents vs. bare metal stents, while acute/subacute stent thrombosis ( $<30$ days $)$ is similar between drug-eluting stents and bare metal stents ${ }^{16}$. In drug-eluting stents, most of the thrombi originate at the flow divider sites where uncovered struts are more frequently observed.

\section{Modalities for local flow assessment in coronary artery bifurcations}

The majority of ESS studies in human coronary bifurcations rely on computational blood flow simulations in three-dimensional (3D) reconstructed models derived from a variety of invasive and noninvasive imaging modalities. 3D quantitative coronary angiography (3D QCA) can provide geometrically accurate representations of coronary bifurcation anatomy and subsequently ESS, as well as fractional flow reserve calculations (Figure 3A) ${ }^{20,21}$. 3D QCA can also be combined with optical coherence tomography (OCT), allowing simultaneous assessment of local flow and plaque features ${ }^{22}$. Fusion of biplane coronary angiography with intravascular ultrasound (3D IVUS) can accurately reproduce the coronary bifurcation geometry and facilitate ESS computation (Figure 3B) ${ }^{8,23}$. 3D OCT is another methodology that has the potential to allow simultaneous assessment of blood flow patterns and plaque and stent morphological features in bifurcation sites (Figure $\mathbf{3 C})^{24}$. There is a lack of large clinical trials of ESS in coronary bifurcations using IVUS or OCT, probably due to the difficulty in interrogating multiple coronary branches.

Non-invasive imaging studies of ESS in bifurcations are based on $3 \mathrm{D}$ reconstruction of arteries using data from cardiac computed tomography (CT). Due to the spatial resolution of CT scanners, small side branches with diameter $<0.5 \mathrm{~mm}$ cannot be accurately reconstructed. ESS studies in bifurcations with the use of CT primarily refer to left main bifurcation ${ }^{25,26}$. Fusion of CT with invasive imaging modalities, such as IVUS, appears to create a fascinating opportunity for $3 \mathrm{D}$ reconstruction and computational simulation of ESS in coronary bifurcations (Figure 3D) ${ }^{8}$. A clinical CT study showed that 


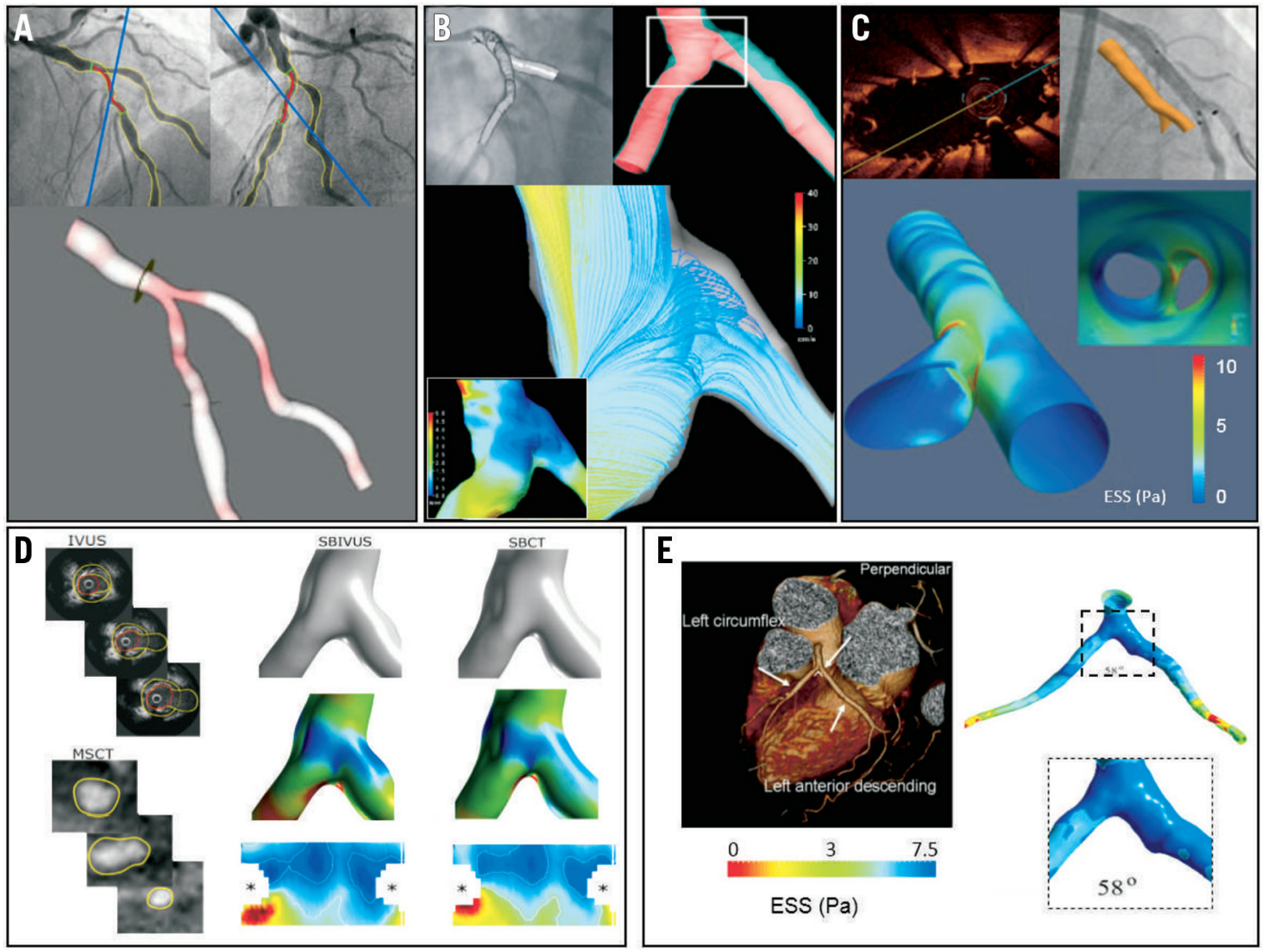

Figure 3. Modalities for flow assessment in coronary bifurcations. A) 3D model of a bifurcation derived from 3D QCA. (Reprinted from Tu et a ${ }^{20}$ with permission from Springer) B) IVUS-based $3 D$ reconstruction and flow simulation of left anterior descending (LAD)-left circumflex (LCx) bifurcation. Low ESS along with slow recirculating flow is demonstrated at the outer wall. (Reprinted from Papafaklis et al ${ }^{23}$ with permission from Elsevier) C) OCT-based $3 D$ in vivo assessment of bifurcation stenting in human. ESS calculation demonstrated high values in the carina and low values at the lateral walls of the bifurcations. (Reprinted from Antoniadis et al ${ }^{24}$ with permission from Oxford University

Press) D) Fusion of IVUS and CT for reconstruction of the side branch in a coronary bifurcation. Representative raw images,

$3 D$ reconstructed bifurcation region without and with the ESS superimposed, and corresponding two-dimensional ESS distribution maps. (Reprinted from Gijsen et al ${ }^{8}$ with permission from Elsevier) E) CT-based, ESS calculation in a single bifurcation and the corresponding bifurcation angle. (Reprinted from Chaichana et al ${ }^{26}$ with permission from Elsevier)

atherosclerotic plaques co-localise with low ESS regions and proposed that the circumferential growth of the plaques from low ESS to high ESS regions might serve as an explanation for the presence of plaques in the high ESS flow divider ${ }^{25}$. Another CT study reported that local ESS and bifurcation angle correlate with left main bifurcation atherosclerosis (Figure 3E) ${ }^{26}$. Improvement of spatial and temporal resolution of cardiac $\mathrm{CT}$ is anticipated to create opportunities for more accurate $3 \mathrm{D}$ reconstructions of the entire coronary tree, leading to patient-specific real-time ESS mapping ${ }^{27}$. Calculation of ESS in coronary artery bifurcations utilising MRI has not been reported to date. The majority of the work in this field is focused on carotid and cerebral artery bifurcations ${ }^{28,29}$. However, recent reports implementing MRI in blood flow measurements in coronary arteries open new perspectives in MRI-based study of ESS in coronary bifurcations ${ }^{30}$.

\section{Coronary stenting and local flow in bifurcations}

In-stent restenosis after stent placement in human coronary arteries has been shown to be associated with low ESS in both bare metal stents $^{31}$ and drug-eluting stents $\mathrm{s}^{32}$. Bifurcation stenting predisposes to restenosis and thrombosis. Adverse local haemodynamics, which are further complicated by the intervention itself, may play a key role in these phenomena ${ }^{16,33}$. Different bifurcation stenting techniques also influence the local flow microenvironment (Figure 4) ${ }^{34}$. In-stent restenosis in the main vessel is more evident in cases of side branch dilation, as compared to the untreated side branch scenario ${ }^{19}$. Concomitant dilation of both the main vessel and side branch might therefore create a restenosis-prone ESS environment that can outweigh the increased cumulative cross-section of the treated bifurcation region ${ }^{19}$. This absence of haemodynamic benefit may account for the lack of apparent clinical benefit observed with side branch angioplasty ${ }^{35}$.

The dimensions, configuration of stent struts and the position of the stent struts with respect to the vessel wall modulate the local haemodynamics, thereby affecting in-stent restenosis, thrombosis and subsequent clinical outcomes ${ }^{36}$. Increased stent strut thickness with less streamlined configuration (i.e., rectangular configuration) is associated with increased rates of in-stent restenosis, probably due 

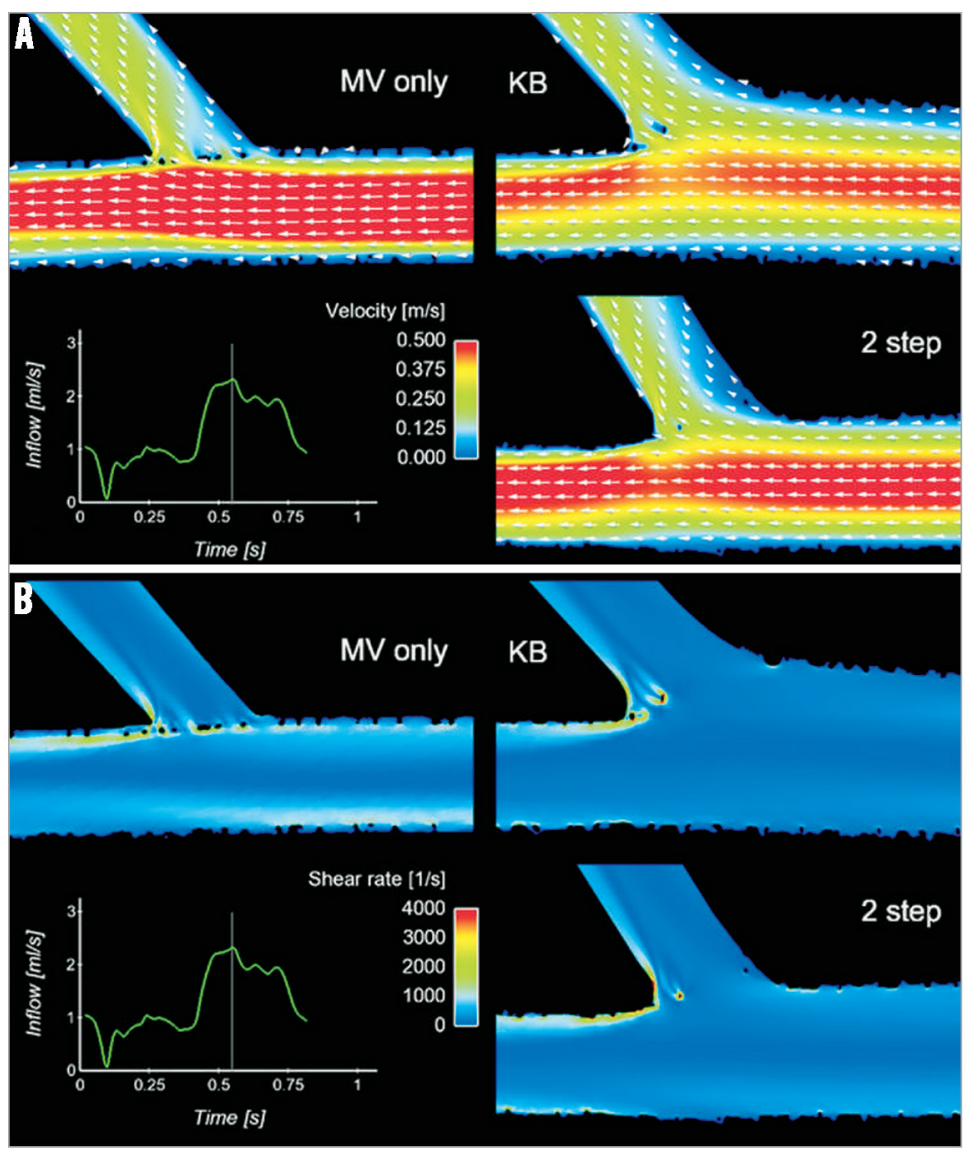

Figure 4. Coronary stenting and local flow in bifurcations. Computational flow simulation of the velocity field (A) and shear rate (B) in cases representative of provisional technique with main vessel (MV) stenting only and post-dilatation with kissing balloon (KB) or the sequential two-step side branch (SB)-MV dilatation. (Reprinted from Foin et al ${ }^{34}$ with permission from Elsevier)

to the generation of high ESS proximal and above the stent struts and low ESS with flow recirculation downstream (distal) to the struts ${ }^{37}$. Malapposition of stents is regarded as a source of increased risk of in-stent restenosis. In-stent restenosis and thrombosis can also occur as a result of stent underexpansion due to the adverse local ESS environment $^{38}$. Stent overlap also creates adverse local haemodynamics which can potentially lead to poor clinical outcomes ${ }^{39}$. From the pathophysiological standpoint, both low and high ESS within stents promote stent restenosis and thrombosis: low ESS upregulates growth factors and modulates local thrombogenicity, whereas high ESS enhances platelet activation and aggregation ${ }^{1,40}$.

\section{Conclusions}

Coronary bifurcation regions exhibit an inherently complex local haemodynamic microenvironment, which subsequently impacts on the localisation, progression and clinical outcomes of plaque formation and treatment. Multiscale computational, animal and human studies have substantially improved our understanding of the interplay between local haemodynamics and the pathophysiology of atherosclerosis in native and stented coronary bifurcations. The advent of advanced invasive and non-invasive imaging modalities is anticipated to facilitate more precise and clinically relevant assessment of geometry and ESS in coronary bifurcations, leading to better clinical outcomes.

\section{Funding}

European Commission, Framework Program 7, Marie Curie International Reintegration Grant, Project: SMILE (249303) to Y. Chatzizisis; Behrakis Foundation, Boston, USA to Y. Chatzizisis and A. Antoniadis; ERC starting grant (BioCCora, 310457) to J. Wentzel.

\section{Conflict of interest statement}

The authors have no conflicts of interest to declare.

\section{References}

The references can be found in the online version of the paper.

\section{Online data supplement}

Online Figure 1. Interplay between coronary anatomy, local ESS microenvironment and vascular biology determines the localisation and complexity of atherosclerosis in coronary bifurcations.

Moving image 1. Blood flow animation in a coronary bifurcation. The proportion of flow directed towards the side branch critically affects the ESS patterns within the bifurcation region. 